\title{
Surgical Hip Dislocation in Symptomatic Cam Femoroacetabular IMPINGEMENT: WHAT MATTERS IN EARLY GOOD RESUlTS?
}

\author{
M. Jäger ${ }^{1 *}$, B. Bittersohl1*, C. Zilkens ${ }^{1}$, H. S. Hosalkar², K. Stefanovska ${ }^{1}$, S. Kurth ${ }^{1}$, R. Krauspe ${ }^{1}$ \\ ${ }^{1}$ Orthopedic Department, Heinrich-Heine University Medical School, Düsseldorf, Germany, \\ ${ }^{2}$ Orthopedic Department, Rady Children's Hospital, San Diego, UCSD, San Diego, CA, USA
}

\begin{abstract}
In order to assess outcome and possible predictors of early good results, a prospective study on 22 patients who were treated with save surgical hip dislocation for symptomatic isolated cam-type femoroacetabular impingement (FAI) was performed. After a follow-up of 6 and 12 months, standard clinical and radiographic parameters were recorded. A statistically significant improvement of the clinical status according to the Harris hip score could be assessed at six months ( $\mathrm{p}-$ value $=0.003)$ and 12 months $(\mathrm{p}$-value $=0.001)$ postsurgery. By comparing standard clinical and radiographic preoperative parameters with various followup outcomes, we revealed no specific parameter with predictive value. These findings are important for centers that have just started to use this surgical technique and are still identifying their learning curve.
\end{abstract}

Key words: hip, FAI, surgery, outcome, predictors

\section{INTRODUCTION}

Femoroacetabular impingement (FAI) that constitutes a repetitive impact of the proximal femur against the acetabular rim is a well known observation in young adults with persisting groin pain and limited range of hip motion [1]. Depending on the underlying mechanism, FAI is further classified as cam-, pincer- or mixed-type. In cam-FAI, the impingement results from an insufficient femoral head-neck offset that may cause cartilage and labrum damage especially during hip flexion. In pincer-FAI, the impingement arises from focal or general acetabular over-coverage probably provoking continuous frontal contact between femoral neck and acetabular labrum during hip flexion. In mixed-FAI, a combination of both mechanisms revealing a damage pattern with combined cam and pincer pathology may be observed.

FAI by itself is not a disease per definition and a variety of factors such as biological tissue integrity remain important. However, emerging evidence has been anticipated that the repetitive abnormal abutment between proximal femur and acetabular rim may eventually lead to early osteoarthritis (OA) of the hip joint [1-3]. In order to possibly stop or delay the onset

* The first and the second author have equally contributed to this study. of the same as well as for managing symptoms, surgical treatment may become necessary [4].

The safe surgical hip dislocation for correction of the morphological abnormality represents a well established technique that proved a low rate of complication and allows for examination and treatment of the entire joint during surgery[2, 5-8]. However, recently only a small number of reports in the English literature can be referred that depict the clinical outcome of this approach in various types of FAI $[2,5,9-12]$ and treatment of various grades of FAI is still a point for discussion - in research meetings as well as in daily clinical practice.

Therefore, our aim in this study was to evaluate the clinical outcome of patients who had undergone surgical hip dislocation and debridement due to symptomatic cam-FAI within a follow-up of one year. We further analyzed preoperative findings in patients with various outcomes after one year in order to reveal valuable predictive factors.

\section{METHODS}

\section{Study Population}

Twenty-two patients with symptomatic cam-FAI that were identified from the orthopaedic outpatient clinic were prospectively studied. There were 15 female $(68.2 \%)$ and 7 male $(31.8 \%)$ patients. Mean age was 36.3 years \pm 11.4 years (range 19.5 years to 59.5 years). All patients had impingement that was symptomatic and warranted surgical intervention. Cam-FAI was verified by clinical examination and imaging studies including plain radiographs and MRI. Confirmation of diagnosis was performed by two senior orthopaedic surgeons (RK and $\mathrm{MJ}$ ) according to previously reported and well established FAI criteria. There were 14 hip joints $(63.3 \%)$ that involved the right side and eight $(36.4 \%)$ that involved the left side. The degree of osteoarthritis classified by standard Tonnis grades was; in three cases $(13.6 \%)$ Tonnis grade 0 , in 14 cases $(63.6 \%)$ Tonnis grade 1 , and in five cases $(22.7 \%)$ Tonnis grade 2. In this study cohort, no patient revealed Tonnis grade 3 changes as these would prevent patients from joint preserving surgery with a promising outcome. The mean Harris Hip Score (HHS) preoperatively evaluated was $46.6 \pm 18.2$ ranging from 21 to 90 . Exclusion criteria such as any other hip pathology than cam-FAI, previous hip surgery, risks or contraindications for MRI 
and surgical treatment were ruled out. Potential side effects were clarified to the patients and their written informed consent obtained prior to the study. This study was approved by the institutional ethic committee.

\section{Medical History}

During the interview, patients were questioned regarding presence and severity of pain, hip joint function and functional activities. On physical exam, range of motion was measured. These observations were summarized and scored according to the HHS system. Symptomatic FAI was verified clinically by the anterior impingement sign performed in supine position by flexing the hip to 90 degrees during adduction and simultaneous internal rotation. The test was considered positive if it would provoke reproducible sharp groin pain similar to their primary complaint of hip pain. During follow-up visits, HHS assessment was repeated six and 12 months post surgery. The HHS after 12 months was used for grading the clinical outcome post surgery where HHSs between 90 and 100 indicated an excellent outcome, HHSs between 80 and 89 indicated a good outcome, HHSs between 70 and 79 represented fair results, and HHS values below 70 defined a poor outcome.

\section{Plain Radiographic Evaluation}

Plain radiographic evaluation was utilized to assess status and morphology of each hip according to the Tonnis criteria where Tonnis grade 0 indicates no signs of osteoarthritis, Tonnis grade 1 proves increased sclerosis and slight joint space narrowing, Tonnis grade 2 small cysts, moderate joint space narrowing and moderate loss of head sphericity, and Tonnis grade 3 large cyst formation, severe joint space narrowing and severe deformity of the femoral head [13]. Therefore, a standard anterior-posterior radiograph of the pelvis and a lateral (Lauenstein) radiograph of the hip were obtained (Fig. 1). On lateral radiographs, the alpha-angle [14] was measured to quantify the femoral head-neck offset within the femoral head-neck junction. The alpha-angle is defined by a parallel drawn within the femoral neck and a line connecting the center of the femoral head with the point where the contour of the femoral head exceeds the femoral head radius (Fig. 2). Alpha-angles of more than $50^{\circ}$ are likely for an offset limitation within the femoral head-neck junction. In order to reveal any progressing of osteoarthritis following surgery, plain radiographs were obtained after 12 months and the Tonnis grading was repeated.
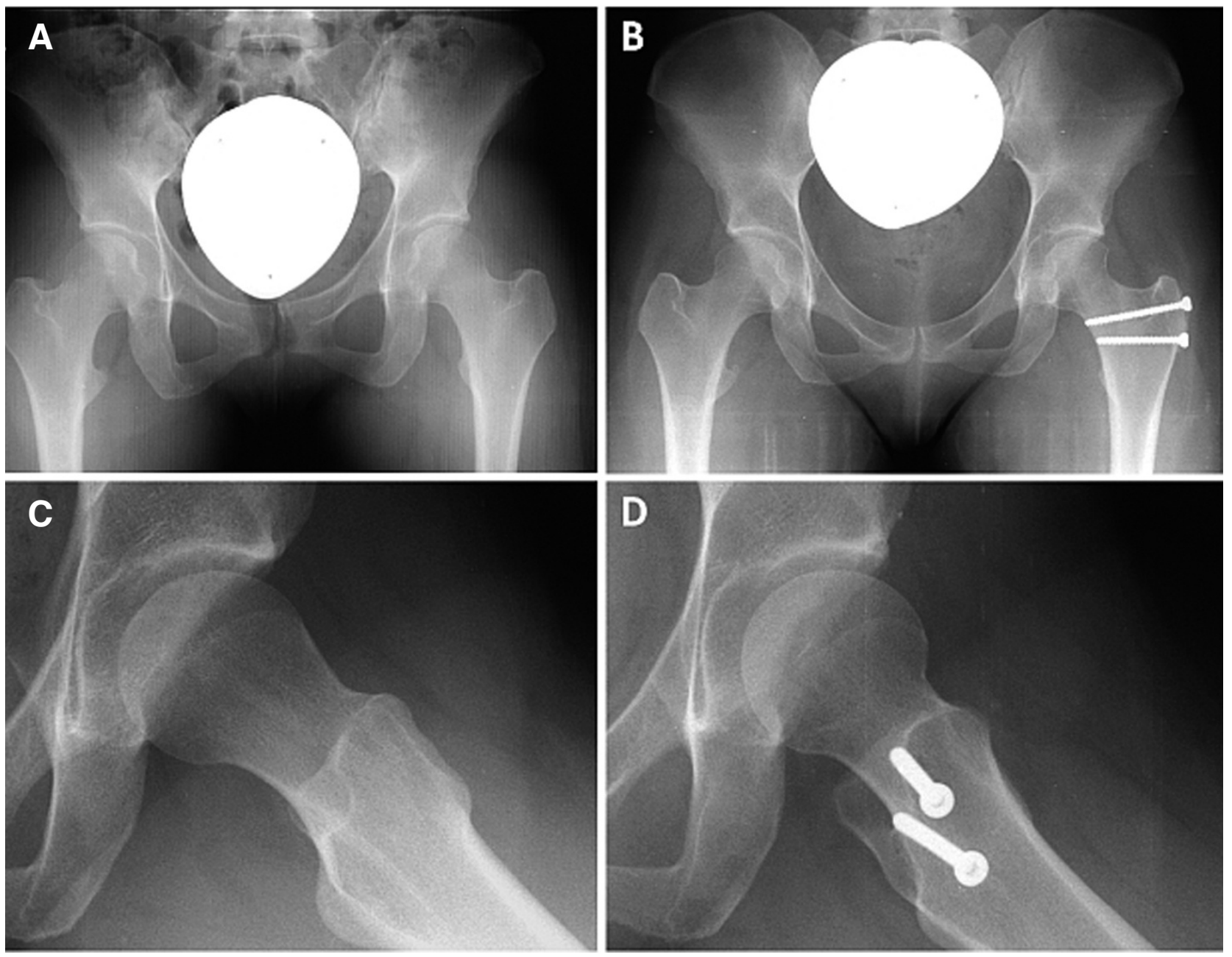

Fig. 1. Standard anterior-posterior radiograph and Lauenstein view pre- (A, C) and post-surgery (B, D). 


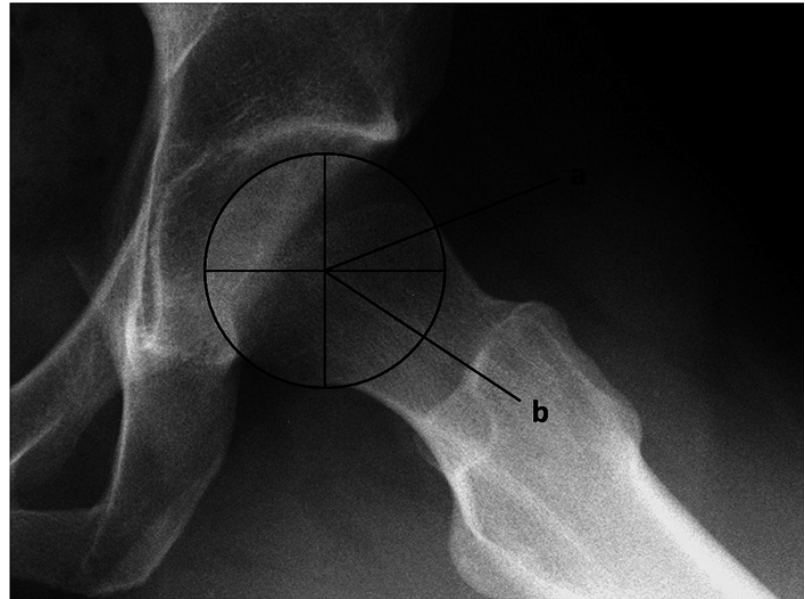

Fig. 2. Alpha-angle assessment within the Lauenstein view. The alpha angle is formed by a parallel drawn within the femoral neck and a line connecting the center of the femoral head with the point where the contour of the femoral head exceeds the femoral head radius.

\section{Magnetic Resonance Imaging (MRI)}

Each patient included in this study underwent MRI with either intraarticular (MR arthrography, MRA) or intravenous contrast agent enhancement (indirect MR arthrography) that was performed in our division or other established MRI departments with equal technical equipment and expertise in hip joint MRI. All MRIs brought to the outpatient visits in our department were evaluated regarding quality and date of acquisition. Each patients included in this present study had appropriate MRI with multidimensional planes and adequate arthrography effect that was undertaken not more than three months before surgery. Within each MR image, femoroacetabular cartilage was assessed in two zones; 1) at the acetabular rim and, 2) at the center of the joint close to the fovea region. It was not possible to differentiate between acetabular and femoral cartilage layers at an image resolution ob-

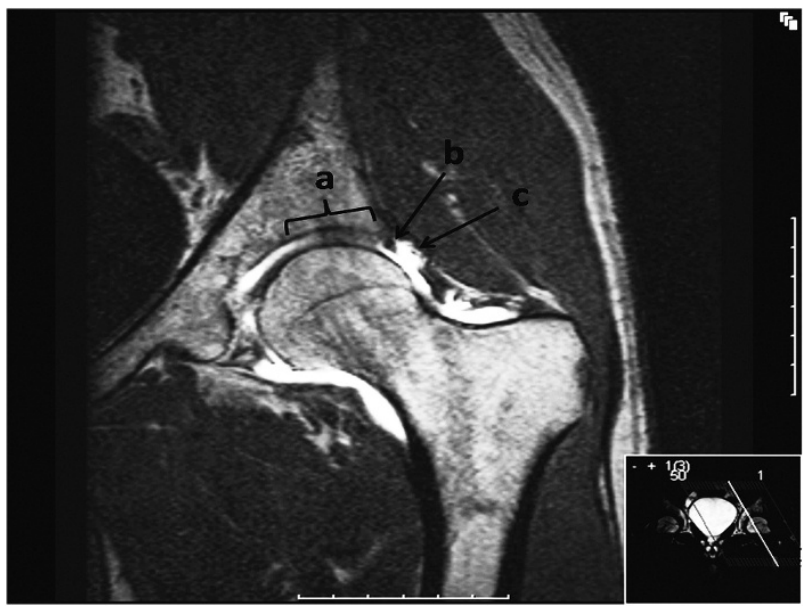

Fig. 3. Oblique sagittal PD-weighted MRA image depicting normal cartilage (a) and normal triangular sharp formation of the labrum without signal alteration (b). The capsule is elevated from the labrum through the contrast medium filling.

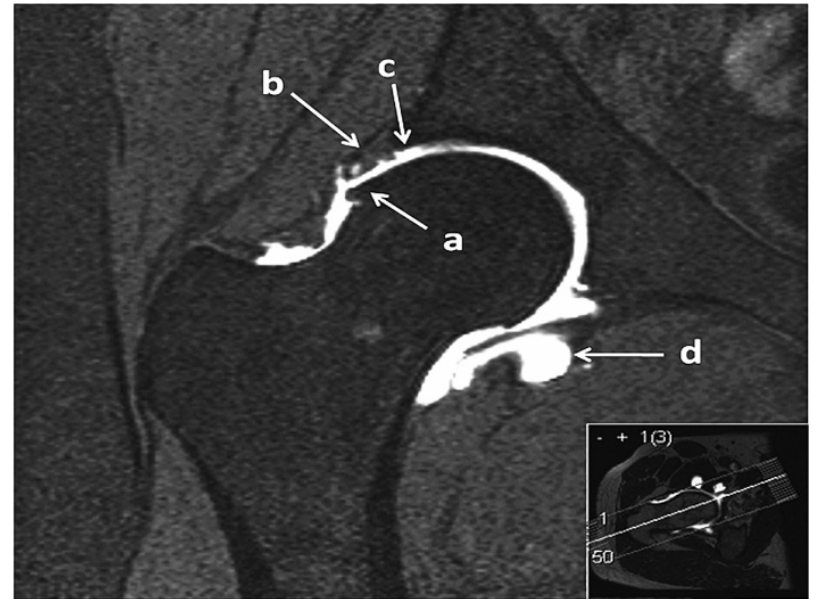

Fig. 4. Oblique coronal T1-weighted MRA image depicting an osseous bump (a), labrum degeneration indicated by labrum hypertrophy and signal alteration (b), severe cartilage degeneration extending medially towards the center of the joint as total cartilage thickness loss at the acetabular rim (c), and contrast medium filling extra-articular (d). Note with MRA, labral tears and cartilage clefts may be better identified through the contrast medium filling into the clefts.

tained with $1.5 \mathrm{~T}$. Therefore, in our study cartilage analysis included acetabular and femoral cartilage as one combined entity. The femoroacetabular cartilage was then evaluated based on the extent of degeneration as used previously [3] for assessment of cartilage damage in FAI where cartilage grade 0 indicated no signs of cartilage degeneration (Fig. 3), cartilage grade 1 indicated degeneration only at the acetabular rim, and cartilage grade 2 represented degeneration extending medially towards the center of the joint (Fig. 4). Cartilage degeneration was defined as any evidence of cartilage changes ranging from signal irregularities to total thickness loss of femoroacetabular cartilage. The acetabular labrum was graded " 0 " indicating a normal formed labrum (Fig. 3), grade 1 indicating signs of degeneration such as signal alteration and/or labrum hypertrophy (Fig. 4), grade 2 depicting a labral tear, and grade 3 indicating the total loss of the labrum.

\section{INTRA-OpERATIVE ANALYSIS}

In all cases surgical intervention included safe surgical hip dislocation in standard technique that represents an approach that allows for inspection of the entire joint surface during surgery (Fig. 5). Hereby, femoroacetabular cartilage and acetabular labrum were evaluated for any signs of degeneration. These findings were clearly documented within a written report and graded according to the MRI classification used in this study. All surgeries and intra-operative evaluations were performed by two senior orthopedic surgeons (RK and MJ) well trained in these procedures.

\section{STATISTICAL ANALYSIS}

Continuous data are provided with mean values and standard deviations (SD) $( \pm)$. To determine whether or not statistically significant difference is present be- 


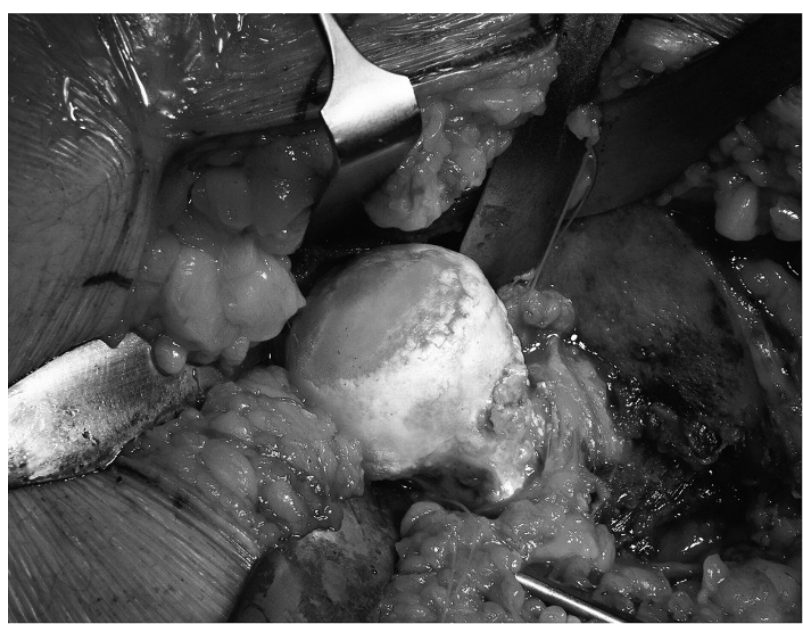

Fig. 5. Intra-operative photograph of the exposed femoral head of patient 1 following safe surgical dislocation demonstrating severe and extensive full thickness cartilage abrasion. Total hip replacement became necessary within 6 months follow-up.

tween preoperative HHSs and follow-up HHSs, the Student's-T-Test (parametric variables, independent samples) was used. In order to reveal existing predictive parameters, we compared preoperative findings in various grades of HHS after 12 months using the Mann-Whitney-U-Test (non-parametric variables, twosided). For correlation analyses, the Spearman-RhoTest (two-sided) was utilized. P-values below 0.05 were considered to be statistically significant. SPSS ${ }$ software (Version 16.0, SPSS Inc., Chicago, IL, USA) was used for all statistical analyses.

\section{RESULTS}

Alpha-angle analyses in this study cohort revealed a considerably increased mean value of $68.9^{\circ} \pm 14.8$ (range $48^{\circ}$ to $96^{\circ}$ ). Mean HHSs were as follows: preoperatively $=46.6 \pm 18.2$ ranging from 21 to 90 , at six months follow-up $=66.2 \pm 21.9$ ranging from 35 to 92 and at 12 months follow up (21 patients included) $=71.9 \pm 20.8$ ranging from 35 to 98 . The increase of HHS during six- and 12 months was statistically significant ( $p$-values $=0.003$ and $<0.001$ ). Comparing the HHSs assessed at six- and at 12 months post surgery this difference did not reach the statistically significance level $(p$-value $=0.522)$. In two cases (patient 17 and patient 22), no increase in the HHS was noted. Here, Tonnis grades of 2 and 1 were evaluated. While in one patient extensive cartilage damage was noted, in the other MRI revealed signal alterations at the acetabular rim while no macroscopic cartilage damage was appearing intra-operatively. Labrum analysis revealed degeneration (patient 17) and controversial findings in patient 22 with a labral tear diagnosed with MRI while no labral damage was noted during surgery. In one case (patient 5) a major drop of the HHS was noted post-surgery that reached from 65 preoperatively to 40 at the 12-month follow-up visit. Interestingly, joint status analysis with radiographic-, MRI and inspection during surgery revealed only slight cartilage changes while a labral tear was noted within the inspection through surgical dislocation. In one patient (patient 14) the good outcome after surgical treatment was only short-termed. While HHS increased from 40 to 89 during a period of 6 months, the same patient proved a HHS of only 35 with similar complains as before he underwent surgery at the 12 months followup. Here, no major cartilage or labrum pathologies have been noted at the beginning of the study. In one further case (patient 1; age at surgery $=32$ years), total hip arthroplasty (THA) became necessary due to progressive OA. Hereby, a Tonnis grade of 1 was observed preoperatively while MRI analyses revealed severe cartilage and labrum damage (grade 2 changes). These observations were confirmed during intra-operative inspection of the hip joint.

Twelve months post-surgery, in six cases $(28.6 \%)$ outcome was classified excellent, three $(14.3 \%)$ were classified good, five $(23.8 \%)$ as fair and seven $(33.3 \%)$ proved poor results. Comparison of preoperative observations based on the 12 month outcome grading revealed no statistically significant difference except for the HHS assessed at six months between patients with excellent outcome and those who proved poor results as well as for labrum observations between the group with excellent outcome and the one with a good outcome although this analysis is limited due to a small number of cases. Statistically significant correlation could be revealed only between the 6-month- and 12month-HHS $(r=0.657$, P-value $=0.001)$.

\section{Discussion}

The safe surgical hip dislocation for deformity correction in FAI is a potential approach that allows for inspection and necessary corrections as trimming of the acetabulum and femoral head-neck region of the entire joint. In this matter, previously reported studies revealed mostly positive results at a short and midterm follow-up [2,5,9-12]. However, with regard to the high number of FAI cases who underwent safe surgical hip dislocation, these study cohorts were relatively small and in some cases inhomogeneous. There is still debate on when to perform surgery and how the outcome may be most likely in various stages of pre-existing damage. Understandably this information is critical in decision making that need to be provided to the patient when discussing diagnosis and subsequent treatment scheme. The aim of this study was therefore to prospectively assess the clinical short-term outcome post-surgery during a follow-up of one year in FAI.

We prospectively performed this study in 22 camFAI patients who proved no other hip deformity than cam-FAI. Subjects with pincer or mixed FAI types were not included. We intended to study the clinical outcome in this particular study cohort and to examine pre- and postoperative observations in order to make out potential factors that may be of value to predict the outcome of this surgical approach. Our results revealed a statistically significant improvement of the clinical status according to the HHSs assessed 6 months ( $\mathrm{p}$-value $=0.003$ ) and 12 months $(\mathrm{p}$-value $=$ 0.001) post-surgery. The difference between the 6month and 12-month HHS was low and did not reach the statistically significance level $(p$-value $=0.522)$ in- 
dicating a notable prognostication degree in the shortterm follow-up. However, a long-term evaluation of this study cohort will have to confirm this observation.

By comparing standard clinical and radiographic preoperative parameters with various follow-up outcomes, we revealed no parameter with predictive value. Correlation between outcome and all parameters assessed preoperatively was weak and not statistically significant. In this study cohort, we noted no increase in the HHS in two cases $(9.1 \%)$. Here, a mixture of findings was observed preoperatively. In one case $(4.6 \%)$ a major drop of the HHS was noted postsurgery. Interestingly, diagnostic assessment with plain radiographs, MRI and inspection during surgery revealed only mild cartilage changes. In one patient (patient $4.6 \%$ ) the good outcome was merely shorttermed. While the HHS increased from 40 to 89 during a period of 6 months, the same patient proved a HHS of only 35 with similar complains as before he underwent surgery at the 12 months follow-up. At the last patient visit 18 months post-surgery, the same patient reached a HHS of 90 (data not provided in this study). In one patient (4.6\%), hip replacement became necessary due to progressive symptoms. Hereby, severe cartilage and labrum damage (Figure 5) was noted. Understandably these observations were based on individual nature and extent of involvement in each subject.

Our results are in keeping with previously reported studies. Beck et al. [5] evaluated the mid-term outcome of FAI patients at a mean follow-up of 4.7 years utilizing the Merle d' Aubigné system that involves a rating of pain, ambulatory status and ROM where a maximum of six points for each subgroup can be reached. In this study cohort, radiographic assessment revealed pistol grip deformity in four and an aspheric shape of the femoral head in seven hips indicating a cam-FAI mechanism. The acetabulum proved abnormal morphological features such as retroversion in five-, coxa profunda in five-, protrusion in three-, and marginal dysplasia in one hip pointing towards a pincer component. A clear differentiation between the various FAItypes was not undertaken. There was a significant improvement ( $p$-value $=0.015)$ in the Merle d'Aubigné score from a preoperative value of 14.1 to 16.5 points at the latest follow-up and the pain score improved statistically significant $(p$-value $=0.005)$ from 2.9 points preoperatively to 5.1 . Interestingly, five out of 19 subjects $(26.3 \%)$ needed THA within the follow-up duration. In the study of 23 FAI patients by Murphy et al.[2] similar results with a statistically significant increase in the Merle d' Aubigné score (13.2 versus 16.9) with seven cases $(30.4 \%)$ where THA became necessary were noted. Hereby, ten patients had isolated cam-FAI, one proved pincer-FAI, and 12 revealed a mixed FAI type. Superior results could be revealed by Espinosa et al.[10] by retrospectively comparing safe surgical hip dislocation for FAI treatment with ( $\mathrm{n}=$ $35)$ and without $(n=25)$ labrum re-fixation. Hereby, a post-operative Merle d'Aubigné score of 17 within the group where the labrum was re-fixated and 15 where no re-fixation was undertaken was noted. The mean preoperative Merle d'Aubigné score in both groups was 12. A clear differentiation between various FAI types in this follow-up study of two years was not performed. Peters and Erickson [12] reported in their study cohort of 29 FAI patients (30 hips) a statistically significant ( $\mathrm{p}$-value $<0.0001$ ) increase of the mean HHS from 70 to 87 within a follow-up of two years. Cam-FAI was diagnosed in 14 hips while pincer-FAI was noted in one case and 15 hips proved radiographic signs indicating a mixed FAI mechanism. The mean follow-up in this study was 32 months. Interestingly, eight hips $(26.7 \%)$ revealed radiographic progression of $\mathrm{OA}$ and four of these eight patients $(13.3 \%)$ needed THA in order to manage symptoms. Similar results were reported by Beaulé at al. [9] in a study of 37 hips in 34 patients with symptomatic cam-FAI where clinical grading scales such as the Western Ontario and McMaster Universities Osteoarthritis Index (WOMAC) at a mean follow-up of 3.1 years. Hereby, nine patients $(20.5 \%)$ underwent screw removal from the greater trochanter because of persistent bursitis and six of the thirty-four patients $(13.6 \%)$ were unsatisfied with the clinical outcome, however, none of the subjects underwent additional hip reconstructive surgery. The authors concluded femoral head-neck offset creation following safe surgical dislocation of the hip joint is an effective approach in cam-FAI treatment and may in most cases improve the overall quality of life. Graves and Mast [11] revealed in their retrospective study a significant increase of the Merle D'Aubigne score from a preoperatively assessed value of 13 to a post-operative score of 16.8 and postulated that surgical dislocation improves symptoms in patients with limited radiographic signs of arthritis who are experiencing impingement-related hip pain. The study included 36 cam- and 12 pincer-FAI hips. Further studies on the outcome of FAI treatment using arthroscopic [15-21] or arthroscopically assisted techniques [22] are published with similar promising results that may be a potential alternative in cases with minor morphological abnormality and less damage in particular in cam-FAI but may be limited in cases where major pathological morphology and degeneration is present.

This study has limitations. First, our study population with 22 cam-FAI patients is relatively small. Further studies should involve a larger study population and various types of FAI. Second, in few cases preoperative cartilage grading was based on indirect MR arthrography which may be less effective compared to direct arthrography (MRA). Furthermore, in all cases MRI was performed utilizing a $1.5 \mathrm{~T}$ system. Therefore, clear differentiation between acetabular and femoral cartilage was not possible and thus cartilage analysis included acetabular and femoral cartilage as one combined entity. Consequently, we may have missed lesions, which could be better identified with MRA and/or 3T MR systems. Third, we performed this study using current standards in FAI diagnosis whereas advanced tools for hip joint diagnosis could be reported recently that may be valuable in the decision making progress in choosing the right therapy approach at the right time [3,23-25].

In summary, the benefit from safe surgical dislocation in this present study of symptomatic cam-FAI pa- 
tients was statistically significant and was in keeping with previously reported studies. None of the standard parameters assessed in this study revealed statistical significance to predict the outcome of this surgical approach in cam-FAI treatment after one year. Further studies are needed to better understand the pathological mechanism of FAI in order to improve the staging of FAI - for 1) early diagnosis of hip joint damage, 2) planning of the appropriate therapy at the right time, and 3) post-operative follow-up.

Acknowledgments: This study was supported (in parts) by a research grant of the "German osteoarthritis aid" ("Deutsche Arthrose-Hilfe e.V.”).

Conflict of interest: The authors declare that they have no conflict of interest.

\section{REFERENCES}

1. Ganz R, Parvizi J, Beck M, Leunig M, Notzli H, Siebenrock KA. Femoroacetabular impingement: a cause for osteoarthritis of the hip. Clin Orthop Relat Res. 2003 Dec; 417): $112-20$

2. Murphy S, Tannast M, Kim YJ, Buly R, Millis MB. Debridement of the adult hip for femoroacetabular impingement: indications and preliminary clinical results. Clin Orthop Relat Res. 2004 Dec; 429): 178-81

3. Bittersohl B, Steppacher S, Haamberg T, Kim YJ, Werlen S, Beck M, Siebenrock KA, Mamisch TC. Cartilage damage in femoroacetabular impingement $(\mathrm{FAI})$ : preliminary results on comparison of standard diagnostic vs delayed gadolinium-enhanced magnetic resonance imaging of cartilage (dGEMRIC). Osteoarthritis Cartilage. 2009 May 3;

4. Tanzer M, Noiseux N. Osseous abnormalities and early osteoarthritis: the role of hip impingement. Clin Orthop Relat Res. 2004 Dec; 429): 170-7

5. Beck M, Leunig M, Parvizi J, Boutier V, Wyss D, Ganz R. Anterior femoroacetabular impingement: part II. Midterm results of surgical treatment. Clin Orthop Relat Res. 2004 Jan; 418): 67-73

6. Spencer S, Millis MB, Kim YJ. Early results of treatment of hip impingement syndrome in slipped capital femoral epiphysis and pistol grip deformity of the femoral headneck junction using the surgical dislocation technique. J Pediatr Orthop. 2006 May-Jun; 26(3): 281-5

7. Ganz R, Gill TJ, Gautier E, Ganz K, Krugel N, Berlemann U. Surgical dislocation of the adult hip a technique with full access to the femoral head and acetabulum without the risk of avascular necrosis. J Bone Joint Surg Br. 2001 Nov; 83(8): 1119-24

8. Lavigne M, Parvizi J, Beck M, Siebenrock KA, Ganz R, Leunig M. Anterior femoroacetabular impingement: part I. Techniques of joint preserving surgery. Clin Orthop Relat Res. 2004 Jan; 418): 61-6

9. Beaule PE, Le Duff MJ, Zaragoza E. Quality of life following femoral head-neck osteochondroplasty for femoroacetabular impingement. J Bone Joint Surg Am. 2007 Apr; 89(4): 773-9

10. Espinosa N, Rothenfluh DA, Beck M, Ganz R, Leunig M. Treatment of femoro-acetabular impingement: preliminary results of labral refixation. J Bone Joint Surg Am. 2006 May; 88(5): 925-35

11. Graves ML, Mast JW. Femoroacetabular impingement: do outcomes reliably improve with surgical dislocations? Clin Orthop Relat Res. 2009 Mar; 467(3): 717-23

12. Peters CL, Erickson JA. Treatment of femoro-acetabular impingement with surgical dislocation and debridement in young adults. J Bone Joint Surg Am. 2006 Aug; 88(8): $1735-41$
13. Tonnis D (1987) Congenital Dysplasia and Dislocation of the Hip in Children and Adults. New York: Springer,

14. Notzli HP, Wyss TF, Stoecklin CH, Schmid MR, Treiber $\mathrm{K}$, Hodler J. The contour of the femoral head-neck junction as a predictor for the risk of anterior impingement. J Bone Joint Surg Br. 2002 May; 84(4): 556-60

15. Bardakos NV, Vasconcelos JC, Villar RN. Early outcome of hip arthroscopy for femoroacetabular impingement: the role of femoral osteoplasty in symptomatic improvement. J Bone Joint Surg Br. 2008 Dec; 90(12): 1570-5

16. Brunner A, Horisberger M, Herzog RF. Sports and recreation activity of patients with femoroacetabular impingement before and after arthroscopic osteoplasty. Am J Sports Med. 2009 May; 37(5): 917-22

17. Byrd JW, Jones KS. Arthroscopic femoroplasty in the management of cam-type femoroacetabular impingement. Clin Orthop Relat Res. 2009 Mar; 467(3): 739-46

18. Ilizaliturri VM, Jr. Complications of arthroscopic femoroacetabular impingement treatment: a review. Clin Orthop Relat Res. 2009 Mar; 467(3): 760-8

19. Ilizaliturri VM, Jr., Orozco-Rodriguez L, Acosta-Rodriguez E, Camacho-Galindo J. Arthroscopic treatment of cam-type femoroacetabular impingement: preliminary report at 2 years minimum follow-up. J Arthroplasty. 2008 Feb; 23(2): 226-34

20. Philippon MJ, Briggs KK, Yen YM, Kuppersmith DA. Outcomes following hip arthroscopy for femoroacetabular impingement with associated chondrolabral dysfunction: minimum two-year follow-up. J Bone Joint Surg Br. 2009 Jan; 91(1): 16-23

21. Stahelin L, Stahelin T, Jolles BM, Herzog RF. Arthroscopic offset restoration in femoroacetabular cam impingement: accuracy and early clinical outcome. Arthroscopy. 2008 Jan; 24(1): 51-7 e1

22. Laude F, Sariali E, Nogier A. Femoroacetabular impingement treatment using arthroscopy and anterior approach. Clin Orthop Relat Res. 2009 Mar; 467(3): 747-52

23. Bittersohl B, Hosalkar HS, Hughes T, Kim YJ, Werlen S, Siebenrock KA, Mamisch TC. Feasibility of T(2) (*) mapping for the evaluation of hip joint cartilage at $1.5 \mathrm{~T}$ using a three-dimensional (3D), gradient-echo (GRE) sequence: A prospective study. Magn Reson Med. 2009 Jul 30;

24. Cunningham T, Jessel R, Zurakowski D, Millis MB, Kim YJ. Delayed gadolinium-enhanced magnetic resonance imaging of cartilage to predict early failure of Bernese periacetabular osteotomy for hip dysplasia. J Bone Joint Surg Am. 2006 Jul; 88(7): 1540-8

25. Kim YJ, Jaramillo D, Millis MB, Gray ML, Burstein D. Assessment of early osteoarthritis in hip dysplasia with delayed gadolinium-enhanced magnetic resonance imaging of cartilage. J Bone Joint Surg Am. 2003 Oct; 85A(10): 1987-92

Received: April 30, 2010 / Accepted: July 15, 2010

Address for correspondence:

Christoph Zilkens MD

Department of Orthopedics,

Heinrich-Heine University Medical School

Moorenstrasse 5

40225 Düsseldorf

Germany

Phone: +49(0)2118117961

Fax: $\quad+49(0) 2118116281$

E-mail: Christoph.Zilkens@med.uni-duesseldorf.de 